\title{
EDITORIALS
}

\section{The Search for More Clinically Meaningful Research Designs:}

\author{
Single-patient Randomized Clinical Trials
}

Clinical Research METHODS often seem too rigid to capture the many subtleties of human illnesses; therefore, innovative, clinically meaningful study designs are always welcome. Earlier this year, researchers at McMaster University brought attention to randomized clinical trials in which two treatments are sequentially tested in a single patient. Publication of two articles in the spring of 1986, one in The Lancet ${ }^{1}$ and another in The New England Journal of Medicine," was followed by an enthusiastic editorial in The Lancet. ${ }^{3}$

Single-patient, ("N of $\mathrm{l}^{\text {") }}$ trials are conducted by systematically varying the management of a patient's illness during a series of treatment periods. In each period the patient sequentially receives one of two treatments; the order is allocated at random. A suitable end-point is negotiated with the patient, and can include a symptom, a sign, a test result, or a patient's preference. ${ }^{1.2}$ Double-blind or other levels of masking ${ }^{4}$ can be established. Several courses of the two treatments are usually necessary for a statistically significant effect to emerge.

A simple example of the design may be the treatment of a hypertensive patient, as follows: period 1, first 3 weeks, hydrochlorothiazide; weeks 4 to 6, hydrochlorothiazide and atenolol; period 2, weeks 7 to 9, hydrochlorothiazide and atenolol; weeks 10 to 12 , hydrochlorothiazide; and so on (the order of treatments is allocated at random).

The approach has a strong appeal to those responsible for patient care, particularly in two common clinical situations: 1) when no sound evidence is available from other studies; and 2) when we disagree with the patient about the effects the treatment is having on him or her. Other circumstances in which the design can be considered include: 3 ) when we attempt to alleviate a (chronic) condition rather than to cure it; 4 ) when the intervention to be tested can provide short-term symptomatic relief; 5) when the intervention effects cease shortly after its withdrawal; 6) when the condition is relatively stable (if a disorder causes "attacks," e.g., epilepsy, angina, migraine, asthma, it is better to rely on long periods of observation and large numbers of patients to reduce variance); 7) when there is a large variability in responses from patient to patient; and 8) when-it is not ethical or feasible to include the patient in a clinical trial.

"N of 1" designs need to be distinguished from other kinds of within-patient or self-controlled studies (those where the patient serves as his own control). Before-after comparisons involve $\alpha$ single intervention, which is tested by comparison of patient status before and after a single treatment. " $\mathrm{N}$ of 1 " studies also differ from the simultaneous comparison of different treatments applied to different body parts of the same patient (e.g., each of the eyes or arms). ${ }^{7}$ Different, too, is the changeover design, which involves multiple crossovers of more than two treatments. ${ }^{8}$ Clinicians planning singlepatient trials should first become familiar with the nuts and bolts of self-controlled studies, so that errors common in these, ${ }^{6.9}$ are not repeated in the apparently straightforward " $\mathrm{N}$ of 1 " designs. Also, much could probably be learned from the behavioral scientists who have used this approach before. ${ }^{1,2}$

The pragmatic or management-oriented (rather than explanatory ${ }^{10}$ ) nature of single-patient trials, as well as some of their advantages and limitations, are adequately described in the Lancet editorial. ${ }^{3}$ However, more emphasis should be given to the similarities between the architecture of " $N$ of 1 " trials and the classic crossover design. ${ }^{11-22}$ Single-case crossover experiments are particularly subject to two sources of bias, sometimes referred to under the common concept of "treatment-period interaction": 1) carryover effects (a pharmacologic effect persists in the second period), and 2) period effects (responses in the second period differ substantially from those in the first period, possibly due to saturation of some biological response, to fluctuations in severity of disease, to patients responding differently because of the "practice" of period 1 , or to other changes in environmental circumstances. $)^{6.11}$

In classic crossover studies, tests for carryover and period effects should routinely be conducted 
before performing the paired $t$ test for treatment differences. ${ }^{23} \mathrm{~A}$ washout phase, during which patients do not receive either of the treatments (or $a$ placebo), can minimize the risk of carryover effects. The use of washout phases and statistical tests for treatment-period interaction in single-patient trials was not discussed with enough detail in the articles mentioned,,$^{1-3}$ and clearly needs further study. Investigators should also keep in mind the problem of regression to the mean, another reason why using patients as their own controls can be misleading. ${ }^{24}$

Pooling results of different single-patient trials on the same intervention or condition will also require careful consideration.

The McMaster team articles emphasize that single-patient trials aim to "determine the most effective treatment for an individual patient," that their "primary purpose . . . is to determine whether the treatment is beneficial in a particular patient," and that they apply "only to the patients who participate in them" [italics added]. These points are well taken because only clinical trials with large sample sizes can be extrapolated to the populations they represent without further testing.

In spite of these warnings, I wonder how often results of " $\mathrm{N}$ of $\mathrm{l}$ " trials will be improperly generalized to other patients. These may in fact be the only designs in which generalization is always inappropriate. Thus, one could ask whether singlepatient trials - while useful to individual patients and physicians - should be published at all. More experience is needed before we can establish when

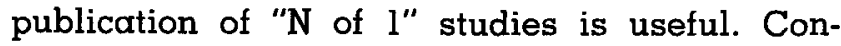
versely, one could argue that any individual case report should try to conform to the single-patient trial rules as closely as possible. Editors could give priority to reports based on this formal design, whenever applicable.

Clinicians who wish to conduct these studies will need a significant amount of time to conduct them (in the examples published, studies took from one to five months). In addition, they will need support in two areas: preparation of active medications (and placebos, when appropriate), and statistical analysis (while common $t$ and Wilcoxon tests will sometimes be applicable, more sophisticated techniques will often be warranted). Clinicians should always consider first to undertake largesomple (crossover or parallel) trials, for which single-patient trials are no substitute. ${ }^{25}$

Single-patient crossover experiments simply formalize a common medical practice: the alternative use and assessment of different therapies in an individual patient. To the extent that they can help couple scientific rigor with clinical relevance, these designs represent a promising re-discovery. The importance of their contributions to dayto-day patient care remains an open question. Miquel S. Porta, MD, MPH, Adjunct Assistant Professor, University of North Carolina School of Public Health; Director, Clinical Epidemiology Unit, Institut Municipal d'Investigació Mèdica (IMIM), Pg. Maritim 25-29, E-08003 Barcelona, Spain

\section{REFERENCES}

1. McLeod RS, Taylor DW, Cohen Z, Cullen JB. Single-patient randomised clinical trial. Use in determining optimum treatment for patient with inflammation of Kock continent ileostomy reservoir. Lancet 1986;1:7268

2. Guyatt G, Sackett D, Taylor DW, Chong J, Roberts R, Pugsley S. Determining optimal therapy - randomized trials in individual patients. N Engl J Med 1986;314:889-92

3. Anonymous. Single-patient trials (editorial). Lancet 1986;1:1254-5

4. Meinert CL. Clinical trials. Design, conduct, and analysis. New York: Oxford University Press, 1986;68-70, 97-100, 200

5. Sackett DL, Haynes B, Tugwell P. Clinical epidemiology: a basic science for clinical medicine. Boston, MA: Little, Brown and Co., 1985; 237$238,180,190,178$

6. Louis TH, Lavori PW, Bailar JC, Polansky M. Crossover and self-controlled designs in clinical research. N Engl 3 Med 1984;310:24-31

7. Pocock SJ. Clinical trials. A practical approach. Chapter 8: Crossover trials. New York: John Wiley \& Sons, 1984;110-122

8. Jesdinsky HJ. Warning against poor application of statistical tests: Confusing experimental intervention and time trends (editorial). Eur $\mathrm{J}$ Clin Pharmacol 1986:30:131-2

9. Cleophas TJM. Statistical concepts fundamental to investigations (letter). N Engl J Med 1985;313:1026

10. Schwartz D, Flammant R, Lellouch J. L'essai therapeutique chez l'homme. 2nd ed. Paris: Flammarion, 1981 [The reference for the English translation of the first (1970) edition is: Same authors. Clinical trials. London: Academic Press, 1980]

11. Hills M. Armitage P. The two-period cross-over clinical trial. Br J Clin Pharmacol 1979;8:7-20

12. Armitage P. Hills M. The two-period crossover trial. Statistician 1982;31:119-31

13. Brown BW Jr. The crossover experiment for clinical trials. Biometrics 1980;36:69-79

14. Vere DW. Validity of cross-over trials (editorial). Br J Clin Pharmacol 1979:8:5-6

15. Anonymous. Planning a cross-over trial (editorial). Lancet 1979;2:511

16. Schwartz D, Flammant R, Lellouch J. L'essai therapeutique chez l'homme. 2nd ed. Paris: Flammarion, 1981;138-44, 153-7

17. Armitage P. Statistical methods in medical research. Oxford: Blackwell. 1980;116-126

18. Zimmermann $\mathrm{H}$, Rahlfs $\mathrm{V}$. Model building and testing for the changeover design. Biometric J 1980;22:197-210

19. Wallenstein S. Fisher AC. The analysis of the two-period repeated measurements crossover design with application to clinical trials. Biometrics 1977;33:261-9

20. Koch GG. The use of non-parametric methods in the statistical analysis of the two-period change-over design. Biometrics 1972;28:577-84

21. Grizzle JE. The two-period change-over design and its use in clinical trials. Biometrics 1965;21:467-80

22. Gore SM, Altman DG. Statistics in practice. London: British Medical Journal Publications, 1982;33-4

23. Bailar JC. Statistical concepts fundamental to investigations (letter). N Engl J Med 1985;313:1026

24. Fletcher RH, Fletcher SW, Wagner EH. Clinical epidemiology - the essentials. Baltimore: Williams \& Wilkins, 1982;135-6, 37-9

25. Newcombe RG, Charny MC. Single-patient trials (letter). Lancet 1986;2:42 